\title{
SAW1 is Required for SDSA Double-Strand Break Repair in S. cerevisiae
}

\author{
Graciel Diamante $^{\mathrm{a}, \boldsymbol{\uparrow}}$, Claire Phan ${ }^{\mathrm{a}, \boldsymbol{\Pi}}$, Angie S. Celis ${ }^{\mathrm{a}}$, Jonas Krueger ${ }^{\mathrm{a}}$, Eric P. Kelson ${ }^{\mathrm{a}}$, and \\ Paula L. Fischhaber ${ }^{\mathrm{a}}$ \\ aDepartment of Chemistry and Biochemistry, California State University Northridge, 18111 \\ Nordhoff ST, Northridge, CA 91330-8262, 011-1-818-677-4503
}

\begin{abstract}
$S A W 1$, coding for Saw1, is required for single-strand annealing (SSA) DNA Double-strand Break (DSB) Repair in S. cerevisiae. Saw1 physically associates with Rad1 and Rad52 and recruits the Rad1-Rad10 endonuclease. Herein we show by fluorescence microscopy that $S A W 1$ is similarly required for recruitment of Rad10 to sites of Synthesis-Dependent Strand Annealing (SDSA) and associates with sites of SDSA repair in a manner temporally overlapped with Rad10. The magnitude of induction of colocalized Saw1-CFP/Rad10-YFP/DSB-RFP foci in SDSA is more dramatic in S and G2 phase cells than in M phase, consistent with the known mechanism of SDSA. We observed a substantial fraction of foci in which Rad10 was localized to the repair site without Saw1, but few DSB sites that contained Saw1 without Rad10. Together these data are consistent with a model in which Saw1 recruits Rad1-Rad10 to SDSA sites, possibly even binding as a protein-protein complex, but departs the repair site in advance of Rad1-Rad10.
\end{abstract}

\section{Keywords}

Saw1; Rad10; Double strand break repair; Synthesis dependent strand annealing

\section{Introduction}

DNA double-strand breaks result from numerous forms of DNA damage caused by myriad damaging agents including ionizing radiation and hydroxyl radicals [1]. DSBs can lead to severe chromosomal alterations including chromosome loss, chromosomal translocations and gene deletions [1]. DSB repair pathways are among the most genetically and mechanistically complex forms of repair. Many DSB genes share redundant or overlapping functions and play roles in some pathways but not others [1].

(C) 2014 Elsevier Inc. All rights reserved.

Correspondence to: Paula L. Fischhaber.

IlThese authors contributed equally to this work.

Publisher's Disclaimer: This is a PDF file of an unedited manuscript that has been accepted for publication. As a service to our customers we are providing this early version of the manuscript. The manuscript will undergo copyediting, typesetting, and review of the resulting proof before it is published in its final citable form. Please note that during the production process errors may be discovered which could affect the content, and all legal disclaimers that apply to the journal pertain. 
DSBs occurring in the presence of a sister chromatid or homologous chromosome can be repaired by homologous recombination, which includes the Synthesis Dependent Strand Annealing (SDSA) pathway. This repair mode is initiated by $5^{\prime} \rightarrow 3^{\prime}$ resection of the $5^{\prime}$ ended strand of each DNA duplex lying to either side of the break [1]. Resection is followed by homology searching and strand invasion of the $3^{\prime}$ overhanging end of one unresected strand into a sister chromatid or homologous chromosome, which is facilitated by RPA, Rad51 and Rad52 [2,3]. New DNA synthesis commences at the $3^{\prime}$ end of the invading strand in a template-directed fashion by DNA Polymerase $\delta$ [4]. The Rad51 filament disassembles and the newly synthesized DNA is displaced, reannealing to the other resected end of the DSB which is promoted by Rad52 [5]. The resulting 3' end contains a flap of excess single-stranded DNA, which is hydrolyzed by the structure-specific Rad1-Rad10 endonuclease [6]. The resulting nick is sealed by DNA Ligase giving rise to a conservatively repaired chromosome [7].

Single-strand Annealing (SSA) is an alternate mode of DSB Repair utilized when DSBs form between DNA repeats and does not generally involve a sister chromatid or homologous chromosome during repair unless multiple DSBs are present in the cell $[1,8]$. The broken chromosome ends are also resected in a $5^{\prime} \rightarrow 3^{\prime}$ fashion, but the resulting $3^{\prime}$ single-stranded ends anneal via the complementary DNA bases deriving from DNA repeats. DNA originally located between the DNA repeats becomes two overhanging $3^{\prime}$ flaps, which are removed by Rad1-Rad10 prior to ligation [9]. SSA is therefore a nonconservative mechanism for repair since the region of DNA originally lying between the DNA repeats is permanently lost. DNA repeats appear throughout the yeast genome, especially in ribosomal DNA of the nucleolus, Ty elements and delta sequences $[1,10]$.

In Saccharomyces cerevisiae, SSA requires the $S A W 1$ gene, which was identified in a screen for SSA mutants and found to be epistatic to RAD1, SLX4, MSH2 and RAD52, among others [11]. Saw1 protein associates physically in vitro with Rad1, Msh2, Msh3, Rad52 and Rad51 [11]. ChIP analysis revealed that $S A W 1$ is required to recruit Rad1 to sites of SSA repair, indicating $S A W 1$ function precedes flap trimming by the Rad1-Rad10 endonuclease in SSA [11]. A recent report showed that Saw1 has intrinsic affinity for flap and splayed arm DNAs and that binding of Saw1 to Rad1-Rad10 increases the affinity of Rad1-Rad10 binding to flap DNAs, especially those containing longer $3^{\prime}$ flaps [12]. A purified complex containing Saw1, Rad1 and Rad10 cleaves specific flap DNA structures in vitro [12]. It has not been shown whether SAW1 plays roles in pathways other than SSA. Using a fluorescence microscopy assay, we investigated whether $S A W l$ might also be required in SDSA.

\section{Materials and Methods}

\section{Cloning of yeast strains Saw1-CFP and saw1}

A disruption mutant of SAWI (PF147-35C) was prepared by gene transplacement using a $L E U 2$ marker flanked by the $S A W 1$ promoter and terminator and transformation into strain WPF006-13C. Transformants were selected on Synthetic Complete agar lacking leucine (SC - LEU agar), sequenced and crossed to strain PF025-7A to produce strain PF147-35C used in microscopy experiments. 
The S. cerevisiae SAWI gene was fused in frame at the chromosomal locus with that of cyan fluorescent protein (CFP) by adaptamer mediated PCR and transformation to prepare Cterminally labeled Saw1-CFP in the haploid W303-1A genetic background, similarly to prior work [13]. Transformants were selected on agar plates lacking uracil followed by backselection on agar containing 5-fluoroorotic acid. PCR, fluorescence microscopy, and DNA sequencing confirmed the presence of the CFP tag, which showed in-frame splicing of CFP and no mutations. The resulting strain was crossed with strain PF025-7A to produce strain PF149-21A used in microscopy experiments.

\section{General microscopy}

Cultures for microscopy experiments were propagated in SC medium supplemented with $200 \mathrm{mg} / \mathrm{mL}$ adenine (SC+ade) at $23{ }^{\circ} \mathrm{C}$ and prepared for microscopy as described previously [13]. Microscopy was performed on a Zeiss AxioImager M1 microscope with a PlanApochromat 100x, 1.46 numerical aperture (NA) objective, as described previously [13]. For 11-slice Z-stacks, images were captured at $0.3 \mu \mathrm{m}$ intervals along the Z-axis; 3-slice Zstacks were obtained by imaging only the 3 slices bounding the center focal plane of the cell. Integration times were $800 \mathrm{~ms}$ for Rad10-YFP and $400 \mathrm{~ms}$ for TetR-RFP for 11-slice Zstacks (experiments in which only YFP and RFP chromophores were imaged). Integration times were $800 \mathrm{~ms}$ each for Rad10-YFP, Saw1-CFP and TetR-RFP for 3-slices Z-stacks (experiments in which YFP, CFP and RFP were all being imaged). Foci were counted and classified by inspecting images from each focal plane as previously described [13]. Budded cells containing one nucleus were classified as S/G2. Budded cells containing two nuclei in physical contact with each other were classified as M. All other cells were classified as G1. Each strain was examined by performing 3 independent trials of at least 100 cells each. Graphs report averages of foci counts from three independent trials and were normalized to differing background foci counts from differences in visual acuity between researchers analyzing the data. Statistical comparisons were carried out by determining a paired $t_{\text {calc }}$ according to the NIST/SEMATECH e-handbook of Statistical Methods and then calculating $\mathrm{p}$ from integration of the single tail area beyond the paired $\mathrm{t}_{\text {calc }}$ in a gaussian distribution. “***” indicates $\mathrm{p}<0.001$, “**” indicates $0.001<\mathrm{p}<0.01$, “*” indicates $0.01<\mathrm{p}<0.05$, "n.s." indicates $0.05<$ p. Images were prepared for publication using Photoshop and Illustrator (Adobe Systems, Mountain View, CA).

\section{Induction of DSBs by I-Scel endonuclease}

Strains PF025-7A, PF147-35C and PF149-21A used for DSB induction experiments bear chromosomally integrated copies of the Tetracycline repressor protein fused to RFP (TetRRFP) and 224 copies of the Tetracycline operator (tetO) repressor binding site abutted with one copy of the I-SceI restriction site (Fig. 1A) at the iYER186 intergenic region on chromosome V as described [14]. The site is cut by I-SceI endonuclease with 60-70\% efficiency in asynchronously growing cells [14]. Strains were transformed with plasmid pWJ1320, containing the I-SceI gene under the control of the GAL1 promoter and an ADE2 selectable marker as described [15]. Transformants were plated on SC-ade agar containing $2 \%$ raffinose ( $\mathrm{SC}-$ ade $\mathrm{w} / \mathrm{raff}$ ). Cultures were propagated in $\mathrm{SC}-$ ade $\mathrm{w} / \mathrm{raff}$ liquid medium at $23{ }^{\circ} \mathrm{C}$. Overnight cultures were diluted to $0.1 \mathrm{OD}_{600}$ and incubated $(3 \mathrm{~h})$, induced by adding 
galactose to the medium for a final concentration of $2 \%(\mathrm{w} / \mathrm{v})$ and incubated $(4 \mathrm{~h})$ prior to microscopy.

\section{SSA/SDSA Southern blot assays}

SSA/SDSA assays were conducted as previously described [13]. Strains were cotransformed with the pJF6 reporter plasmid or a derivative containing a HIS3 marker cloned into the SalI site of pJF6 (pJF6-HIS3) and with pFH800, containing the HO endonuclease gene under the control of the GAL1 promoter. Starter cultures were incubated in Synthetic Complete medium lacking leucine and tryptophan (SC-Leu-Trp) for experiments with pJF6, or histidine, leucine and tryptophan (SC-His-Leu-Trp) for pJF6-HIS3, inoculated into YEPLactate and grown to $5 \times 10^{6}$ to $1 \times 10^{7}$ cells $/ \mathrm{mL}$, induced by galactose addition $(2 \%$, w/v) and incubated with shaking (4 h). Plasmid DNA was isolated, digested with HindIII, PstI and $S m a \mathrm{I}$, resolved on a $0.8 \%$ agarose gel (1x TAE, $50 \mathrm{~V}, 21 \mathrm{~h})$, and transferred to Immobilon-Ny+ (Millipore). The membrane was hybridized $\left(12 \mathrm{~h}, 68{ }^{\circ} \mathrm{C}\right)$ with a digoxigenin-labeled (Roche) DNA probe prepared by PCR amplification of an $\sim 800 \mathrm{bp}$ fragment of LacZ using primers $5^{\prime}$-d(CGTCATAGCGATAACGAG) and $5^{\prime}$ d(CGGTCGGGATAGTTTTCTTGCG). The probe was detected using a standard adigoxigenin/CSPD protocol and manufacturer's instructions (Roche). Quantity One image analysis software (BioRad) was used for densitometric calculations.

\section{Results}

\section{Recruitment of Rad10 to SDSA is abrogated in absence of SAW1}

To determine whether Saw1 plays a role in SDSA we prepared a strain of S. cerevisiae deleted of the SAWI gene ( $\operatorname{saw1} \Delta$ ) that also contained a chromosomally integrated, fluorescently labeled copy of the $R A D 10$ gene (Rad10-YFP) and an inducible, fluorescently labeled DSB site not flanked by DNA repeats which is repaired by SDSA [13]. DSB induction experiments with this $\operatorname{saw} 1 \Delta$ strain were carried out in parallel with a corresponding $S A W 1$ wild-type strain (Fig. 1A and B). Data show that in asynchronously growing cells, Rad10-YFP foci colocalization with DSB-RFP foci is induced 2.1 fold in budded cells (S, G2 and M phases, 33\% induced/ 16\% uninduced) while experiments carried out in the saw $1 \Delta$ strain show only background levels of Rad10-YFP/DSB-RFP colocalization in dividing cells, with or without DSB induction (8.0-10\%, Fig. 1B). G1 phase cells showed only background levels of colocalized foci in the presence or absence of DSB induction (4.0-7.1\%), indicating no Rad10-mediated repair was being carried out in G1 (Fig. 1B). These data indicate that Rad10 recruitment requires $S A W 1$.

\section{Saw1-CFP exhibits foci that colocalize to DSB sites with Rad10-YFP in SDSA}

To further investigate the function of $S A W 1$ we prepared a yeast strain containing $S A W 1$ fused to CFP (Saw1-CFP), which exhibited dim pan-nuclear fluorescence indicative of low protein abundance and nuclear localization (Fig. 2A). We tested the behavior of Saw1-CFP in experiments similar to those in Fig. 1. Upon induction of DSBs, we observed colocalized foci containing Rad10-YFP, Saw1-CFP and DSB-RFP (triple colocalized foci) indicating that the functions of Rad10 and Saw1 temporally overlap at SDSA sites (Fig. 2B). 
We counted triple colocalized foci (Rad10-YFP/Saw1-CFP/DSB-RFP), double colocalized foci (Saw1-CFP/DSB-RFP, Rad10-YPF/DSB-RFP and Saw10-CFP/Rad10-YFP) and single Rad10-YFP foci or Saw1-CFP foci. Foci counts were used to estimate abundances of repair sites containing both Rad10 and Saw1, or only Rad10 or Saw1, and locations where Saw1 and Rad10 were colocalized but not at induced DSB sites. Following DSB induction, Rad10-YFP and Saw1-CFP colocalize at DSB-RFP sites in greater abundance than in uninduced controls, especially in S and G2 phase cells (12-fold induction; 0.170 focus per cell induced/0.014 focus per cell uninduced) (Fig. 2C). In M phase cells we observed a modest 0.048 focus per cell in induced samples, but no triple foci in uninduced controls (Fig. 2C). G1 phase cells exhibited modest abundances that were not statistically different between induced and uninduced samples (Fig. 2C). Hence, Saw1 localizes to repair sites in a manner that temporally overlaps Rad10 in budding cells.

Fluorescent images were also examined for Rad10-YFP/DSB-RFP double colocalized foci (i.e. that did not contain Saw1-CFP). S and G2 cells exhibited a 3.8-fold induction of Rad10YFP/DSB-RFP double colocalized foci ( 0.084 focus per cell induced/ 0.022 focus per cell uninduced) (Fig. 2C). M cells exhibited 0.073 Rad10-YFP/DSB-RFP colocalized focus per cell following induction, but no foci in uninduced controls. G1 cells exhibited a 3.8 fold induction ( 0.065 focus per cell induced/ 0.017 focus per cell uninduced). The pattern of induction of Rad10-YFP/DSB-RFP foci indicates that some repair events contain Rad10 but not Saw1 thereby suggesting that the roles of Rad10 and Saw1 are not completely temporally coincident. The induction of triple foci was higher than Rad10-YFP/DSB-RFP double foci (12-fold triple vs. 3.8-fold double) suggesting that in S and G2, duration of occupancy of the DSB site by Saw1 may be a significant fraction ( $250 \%)$ of the duration of occupancy of Rad1-Rad10.

These observations were compared to quantification of Saw1-CFP/DSB-RFP double colocalized foci (i.e that did not contain Rad10-YFP). In all phases of cell cycle we observed only background levels of such foci either with or without induction of DSBs $(0.034$ or fewer focus per cell) (Fig. 2C). This observation of so few Saw1-CFP/DSB-RFP double foci, coupled with our finding that Rad10 cannot be recruited in the absence of $S A W 1$, suggests that Rad1-Rad10 may be recruited to the repair site with Saw1 or, at least, shortly following Saw1. Timelapse experiments were attempted to determine whether there was a temporal offset of the localization of Saw1-CFP and Rad10-YFP to DSB-RFP sites but were hampered by the low abundances of Saw1-CFP and Rad10-YFP as well as photobleaching of both when fields of cells were imaged repeatedly. Together, the data suggest that Saw1 and Rad10 are recruited at the same time or almost the same time and that Saw1 departs the repair site in advance of Rad10.

We also examined fluorescent images for Saw1-CFP/Rad10-YFP foci that were not colocalized with DSB-RFP. S and G2 cells contained a slightly higher number of spontaneous Saw1-CFP/Rad10-YFP foci in uninduced control cells (0.099 focus per cell) which dropped to background levels ( 0.033 focus per cell) upon induction of DSBs (Fig. 2C). Only background levels were observed in M and G1 cells ( 0.034 focus per cell or fewer, induced or uninduced). The results in S and G2 cells suggest a subpopulation of Saw1 and Rad10 are localized with each other at spontaneous DNA damage sites, potentially in a 
multiprotein complex, which then relocalizes to SDSA sites. "Single" foci in which Saw1CFP or Rad10-YFP foci were not colocalized with each other or with DSB-RFP foci were observed at background levels in S/G2 and G1 ( $<0.045$ focus per cell) (Fig. 2D).

Abundances were slightly higher in M phase cells ( 0.070 focus per cell) (Fig. 2D).

\section{Saw1-CFP is a reliable reporter of the behavior of Saw1 in SDSA and SSA}

To ensure that Saw1-CFP protein is functional for DSB Repair, a Southern blot assay was employed. Strains were transformed with a plasmid containing $\mathrm{HO}$ endonuclease under the control of the GAL1 promoter and a reporter plasmid containing two incomplete copies of the $\beta$-gal gene, one containing an $\mathrm{HO}$ restriction site which can be repaired either by SDSA or SSA (Fig. 3A). We observed that a strain containing the Rad10-YFP/DSB induction system and Saw1-CFP exhibits similar levels of SDSA and SSA as a wild-type RAD10 SAWl strain (Fig. 3B, compare $5.8 \mathrm{~kb}$ band intensities in lanes 1 and 5 and $4.3 \mathrm{~kb}$ band intensities in lanes 1 and 5). Control experiments performed with a strain deleted of RAD10 ( rad10A), show no SSA or SDSA products (Fig. 3B, lane 3). Uninduced controls showed no repair products (Fig. 3B, lanes 2, 4 and 6). Densitometric analysis (Fig. 3B, lanes 1, 3 and 5) indicates that the Rad10-YFP/Saw1-CFP strain exhibits the same efficiency of SSA (99\%) and a slightly higher efficiency of SDSA (137\%) as RAD10 SAWl (Fig. 3B). To ensure that $S A W 1$ is required to observe repair in our Southern Assay, the assay was repeated with a saw $1 \Delta$ strain resulting in only background levels of the SDSA product (9\%) and significantly reduced levels of the SSA product (32\%) (Figs. 3D and 3E). Together these results indicate Saw1-CFP functions similarly to wild-type Saw1, and is a faithful fluorescent reporter.

\section{Discussion}

Prior reports showed Saw1 is required to recruit Rad1-Rad10 to SSA sites and that Saw1 increases the affinity of Rad1-Rad10 for flap DNAs in vitro, particularly for longer $3{ }^{\prime}$ flaps $[11,12]$. Herein we report a requirement for SAWI in SDSA by showing explicitly that recruitment of Rad10 to sites of DSBs being repaired by SDSA is abrogated in the absence of SAW1. Further, we show Saw1 localization to SDSA sites is significantly temporally overlapped with Rad10. The temporal overlap between Saw1 and Rad10 at SDSA sites is not entirely coincident as a relatively large percentage of cells contained Rad10 localized to repair sites without Saw1. The converse was not observed, however; very few cells were observed in which Saw1 was localized to repair sites without Rad10. Together these observations show that Saw1 is required to recruit Rad10 but imply that Saw1 departs prior to Rad10. It is formally possible that the triple and double foci in question represent different types of SDSA sites, and the latter never included Saw1, but we do not favor this explanation owing to results showing Rad10 recruitment is abrogated in a sawl $1 \Delta$ background (Fig. 1A).

Our observations also address the temporal relationship between Saw1 and Rad10 at the moment of recruitment. First, we observed few Saw1 foci occupying SDSA sites without Rad10, either in the presence or absence of DSB induction. Second, S and G2 cells, exhibited a significant number of Saw1-CFP/Rad10-YFP colocalized foci in uninduced 
controls that were not localized to DSB-RFP sites. This number dropped upon DSB induction concomitant with increased Saw1 and Rad10 localization to DSB-RFP sites. These results suggest Saw1-Rad1-Rad10 complexes can relocalize from one repair site to another and are consistent with an earlier report showing a tight Saw1-Rad1-Rad10 complex forms in vitro [12]. Together these observations are consistent with Saw1 and Rad10 being recruited together or with very little temporal offset. Since Saw1 interacts physically with both Rad52 and Rad1, Saw1 may mediate recruitment of Rad1-Rad10 to DSB sites via Rad52, occupy the repair site for a short time, and then dissociate prior to $\operatorname{Rad} 1-\operatorname{Rad} 10$.

Our data suggest Saw1 occupancy may be $250 \%$ the duration of occupancy of Rad1-Rad10. We observed nearly twice as many Saw1-CFP/Rad10-YFP/DSB-RFP triple foci as Rad10YFP/DSB-RFP double foci in S/G2 cells and approximately equally as many triple foci as double foci in M cells (Fig. 2C). Interestingly, our previous work showed Rad52 also exhibits considerable temporal overlap with Rad1-Rad10 at SDSA sites, but that numerous repair sites contained Rad1-Rad10 without Rad52 [13]. These observations are consistent with a model in which Saw1 and Rad1-Rad10 are recruited together but in which Rad52 and Saw1 dissociate prior to Rad1-Rad10. A scheme depicting this model is shown (Fig. 4).

Although Saw1-CFP and Rad10-YFP exhibit pan-nuclear fluorescence (Fig. 2B), we did not observe numerous Saw1-CFP/Rad10-YFP double foci throughout the nuclei as a background in our experiments despite prior reports showing Saw1-Rad1-Rad10 complex formation in vitro and that Saw1 is not stable in radl $1 \Delta$ or rad10 $\Delta$ deletion strains [11,12]. It is possible that few background double foci are visible because Saw1-Rad1-Rad10 complexes do not form until bound at repair sites, but we believe it more probable that most Saw1-Rad1-Rad10 complexes are not visible as foci due to high mobility Brownian motion when not engaged in repair. Fluorescence photobleaching experiments have shown that proteins freely diffusing throughout the nucleus become fully redistributed within $50 \mathrm{~ms}$, which is a much shorter timeframe than the $800 \mathrm{~ms}$ exposures used by us for Saw1-CFP and Rad10-YFP [16]. We have proffered here that the relatively abundant Saw1-CFP/Rad10YFP double foci observed in uninduced S/G2 cells (Fig. 2C) may represent Saw1-Rad1Rad10 complexes localized to spontaneous repair sites. It is not clear why these double foci are more abundant in S and G2 cells. Investigation of other possible roles of Saw1 in DSB repair is important in determining the molecular basis of these other foci.

\section{Acknowledgments}

The authors thank Dr. Michael Lisby for helpful comments on the manuscript. This work was supported by NIH grants SC2GM081155 (to PLF), SC3GM093858 (to PLF), S06GM48680 (to EPK) and 5T34GM008395 (to GD).

\section{References}

1. Friedberg, EC.; Walker, GC.; Siede, W.; Wood, RD.; Schultz, RA.; Ellenberger, T. DNA Repair and Mutagenesis. 2. ASM Press; Washington, DC: 2005.

2. New JH, Sugiyama T, Zaitseva E, Kowalczykowski SC. Rad52 protein stimulates DNA strand exchange by Rad51 and replication protein A. Nature. 1998; 391:407-410. [PubMed: 9450760]

3. Sung P. Function of yeast Rad52 protein as a mediator between replication protein A and the Rad51 recombinase. The Journal of biological chemistry. 1997; 272:28194-28197. [PubMed: 9353267] 
4. Maloisel L, Fabre F, Gangloff S. DNA polymerase delta is preferentially recruited during homologous recombination to promote heteroduplex DNA extension. Molecular and cellular biology. 2008; 28:1373-1382. [PubMed: 18086882]

5. Sugiyama T, Kantake N, Wu Y, Kowalczykowski SC. Rad52-mediated DNA annealing after Rad51-mediated DNA strand exchange promotes second ssDNA capture. The EMBO journal. 2006; 25:5539-5548. [PubMed: 17093500]

6. Fishman-Lobell J, Haber JE. Removal of nonhomologous DNA ends in double-strand break recombination: the role of the yeast ultraviolet repair gene RAD1. Science. 1992; 258:480-484. [PubMed: 1411547]

7. Wang X, Ira G, Tercero JA, Holmes AM, Diffley JF, Haber JE. Role of DNA replication proteins in double-strand break-induced recombination in Saccharomyces cerevisiae. Molecular and cellular biology. 2004; 24:6891-6899. [PubMed: 15282291]

8. Pannunzio NR, Manthey GM, Bailis AM. RAD59 is required for efficient repair of simultaneous double-strand breaks resulting in translocations in Saccharomyces cerevisiae. DNA repair. 2008; 7:788-800. [PubMed: 18373960]

9. Sugawara N, Paques F, Colaiacovo M, Haber JE. Role of Saccharomyces cerevisiae Msh2 and Msh3 repair proteins in double-strand break-induced recombination. Proceedings of the National Academy of Sciences of the United States of America. 1997; 94:9214-9219. [PubMed: 9256462]

10. Mieczkowski PA, Lemoine FJ, Petes TD. Recombination between retrotransposons as a source of chromosome rearrangements in the yeast Saccharomyces cerevisiae. DNA Repair (Amst). 2006; 5:1010-1020. [PubMed: 16798113]

11. Li F, Dong J, Pan X, Oum JH, Boeke JD, Lee SE. Microarray-based genetic screen defines SAW1, a gene required for Rad1/Rad10-dependent processing of recombination intermediates. Mol Cell. 2008; 30:325-335. [PubMed: 18471978]

12. Li F, Dong J, Eichmiller R, Holland C, Minca E, Prakash R, Sung P, Yong Shim E, Surtees JA, Eun Lee S. Role of Saw1 in Rad1/Rad10 complex assembly at recombination intermediates in budding yeast. The EMBO journal. 2013; 32:461-472. [PubMed: 23299942]

13. Moore DM, Karlin J, Gonzalez-Barrera S, Mardiros A, Lisby M, Doughty A, Gilley J, Rothstein R, Friedberg EC, Fischhaber PL. Rad10 exhibits lesion-dependent genetic requirements for recruitment to DNA double-strand breaks in Saccharomyces cerevisiae. Nucleic Acids Res. 2009; 37:6429-6438. [PubMed: 19729509]

14. Lisby M, Mortensen UH, Rothstein R. Colocalization of multiple DNA double-strand breaks at a single Rad52 repair centre. Nat Cell Biol. 2003; 5:572-577. [PubMed: 12766777]

15. Lisby M, Barlow JH, Burgess RC, Rothstein R. Choreography of the DNA damage response: spatiotemporal relationships among checkpoint and repair proteins. Cell. 2004; 118:699-713. [PubMed: 15369670]

16. Sprague BL, McNally JG. FRAP analysis of binding: proper and fitting. Trends in cell biology. 2005; 15:84-91. [PubMed: 15695095]

17. Thomas BJ, Rothstein R. Elevated recombination rates in transcriptionally active DNA. Cell. 1989; 56:619-630. [PubMed: 2645056]

18. Mardiros A, Benoun JM, Haughton R, Baxter K, Kelson EP, Fischhaber PL. Rad10-YFP focus induction in response to UV depends on RAD14 in yeast. Acta histochemica. 2011; 113:409-415. [PubMed: 20546858] 


\section{EES Highlights}

- Saw1 is required for Synthesis Dependent Strand Annealing in S. cerevisiae.

- Saw1 foci are temporally overlapped with Rad10 foci at SDSA sites.

- Few Saw1 foci appear at SDSA sites without Rad10.

- Temporal overlap of Saw1 and Rad10 foci at SDSA sites is not entirely coincident. 
A.

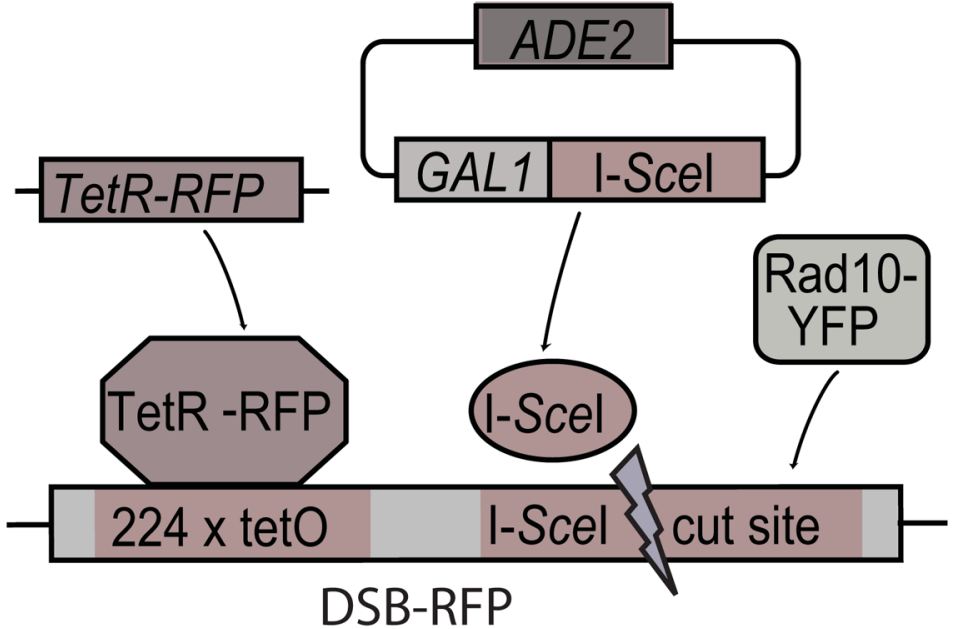

\section{B. Rad10-YFP/DSB-RFP Colocalized Foci}

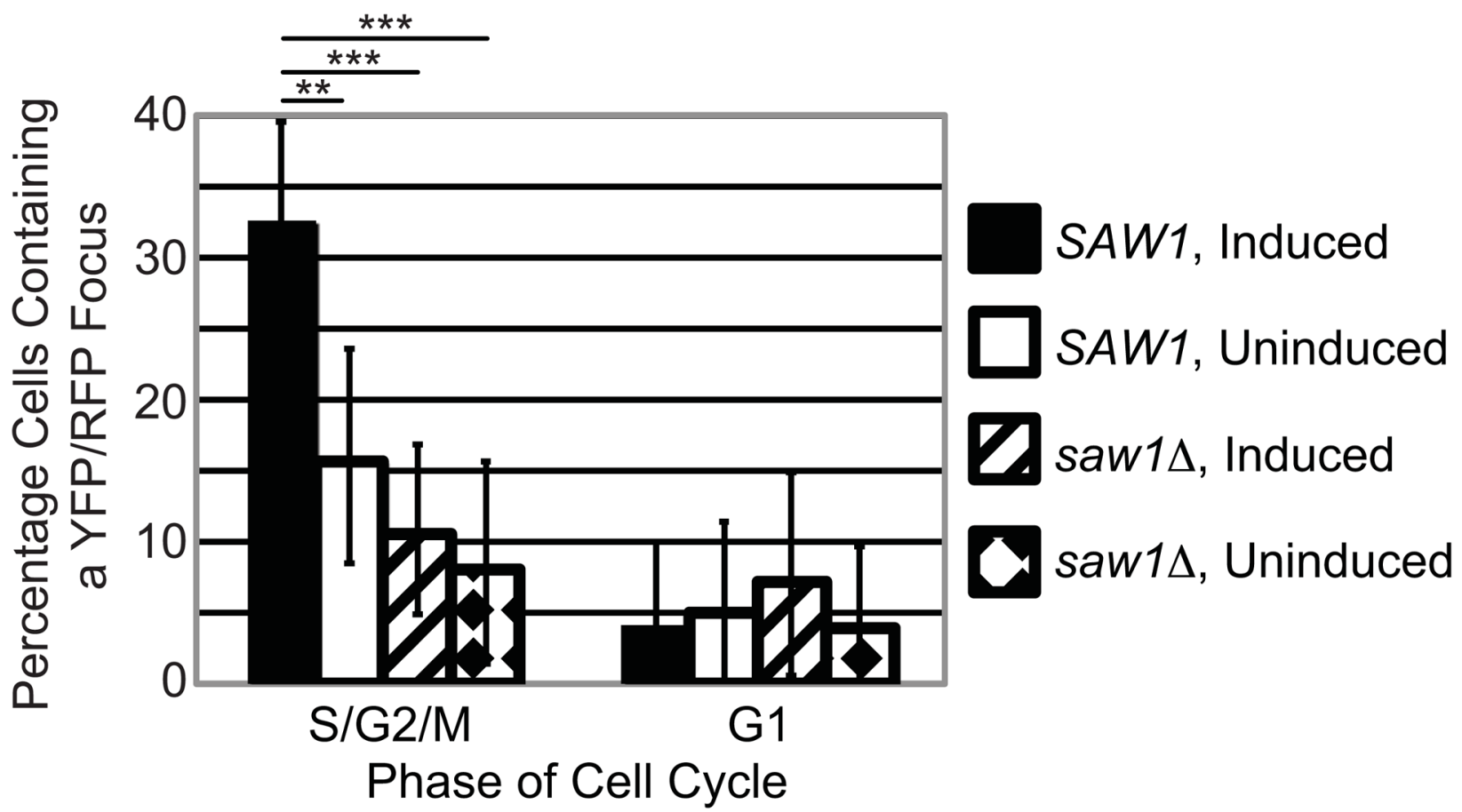

Figure 1.

Saw1 is required for SDSA. A. Illustration of the fluorescence assay system. Yeast strains containing TetR-RFP and Rad10-YFP are transformed with plasmid pWJ1320, harboring an ADE2 selection marker and the I-SceI gene under control of the GAL1 promoter. TetR-RFP protein binds tetO sequences adjacent to the I-SceI restriction site, inducing a DSB near TetR-RFP (DSBRFP). Recruitment of Rad10-YFP protein to the DSB-RFP site is monitored. B. Graph of YFP/RFP colocalized foci counts. Statistical comparisons were carried out as described in Materials and Methods. SAW1 (PF025-7A; black bars = galactose induction; white bars = uninduced control $)$ and saw $1 \Delta(\mathrm{PF} 147-35 \mathrm{C}$; striped bars = galactose induction; stippled bars = uninduced control). 
A.

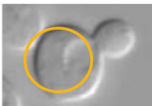

DIC

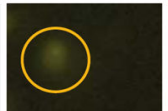

YFP

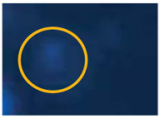

CFP

C. Colocalization of Saw1-CFP, Rad10-YFP and DSB-RFP Foci

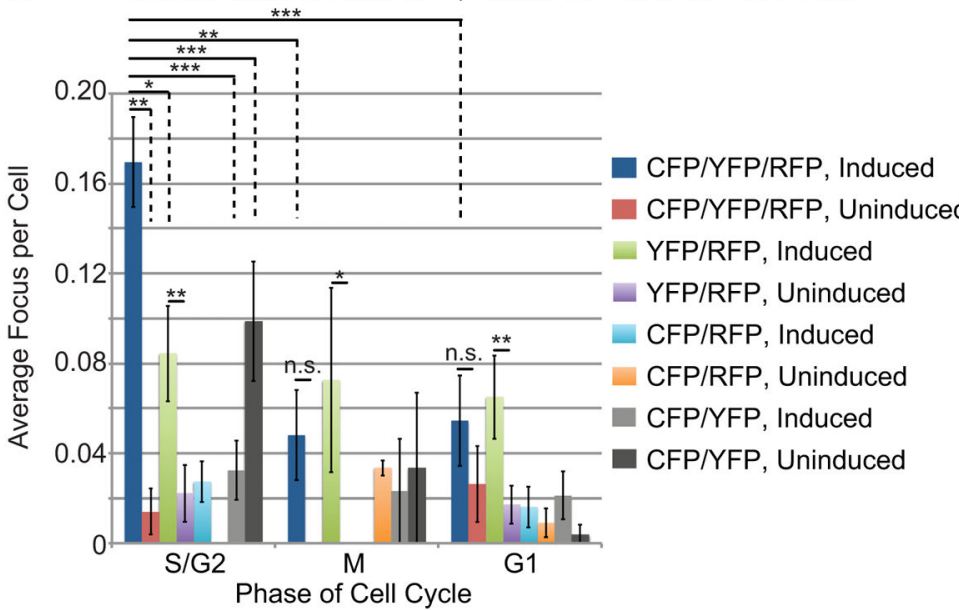

B.

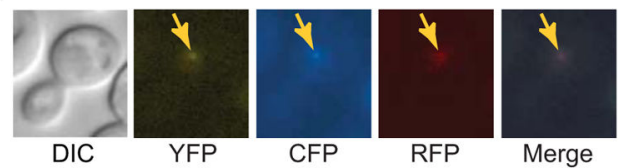

D. Single Saw1-CFP \& Rad10-YFP Foci

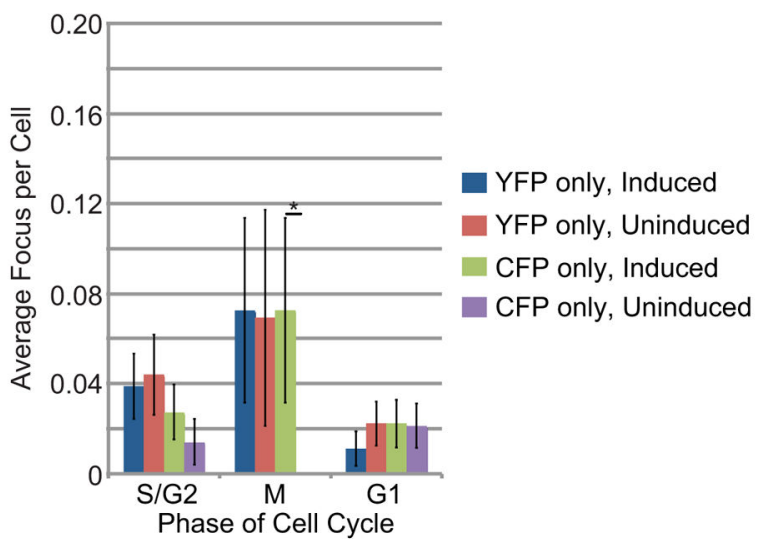

Figure 2.

Saw1 colocalizes to SDSA repair sites. A. Saw1-CFP exhibits pan-nuclear fluorescence. Images show strain PF149-21A; DIC = differential interference contrast, $\mathrm{YFP}=$ Yellow fluorescence, $\mathrm{CFP}=$ cyan fluorescence. A yellow circle indicates the nucleus. $\mathrm{B}$. Saw1-CFP and Rad10-YFP colocalize to SDSA sites. Images show strain PF149-21A transformed with pWJ1320 and DSBinduced by galactose. Yellow arrows indicate a focus containing Rad10-YFP, Saw1-CFP and DSB-RFP. DIC, YFP, CFP and RFP images are shown along with an overlay of the latter three (YFP/CFP/RFP). C. Saw1-CFP and Rad10-YFP colocalize to sites of SDSA in high abundance. Focus counts from DSB induction experiments in strain PF149-21A transformed with pWJ1320. Graph categorizes foci according to the highest ordering of fluorescent markers present (Rad10-YFP, Saw1-CFP, DSB-RFP). For example, a triple focus containing YFP, CFP and RFP was classified as a "CFP/YFP/RFP" focus and does not appear in the calculations for CFP/YFP, YFP/RFP or CFP/RFP foci. "Induced" = galactose induction; "Uninduced" = uninduced control. D. Same experiment as C but showing abundances of uncolocalized CFP and YFP foci observed. DSB-RFP foci abundances were not plotted since nearly every cell exhibits such a focus whether or not undergoing repair. Plotted values represent means ( $\lambda$ 's) from ideal Poisson distributions least squares fit to the observed distributions of foci per cell. The least squares deviations were propagated into the overall errors, which are depicted as the error bars. Statistical comparisons were carried out as described in Materials and Methods. 

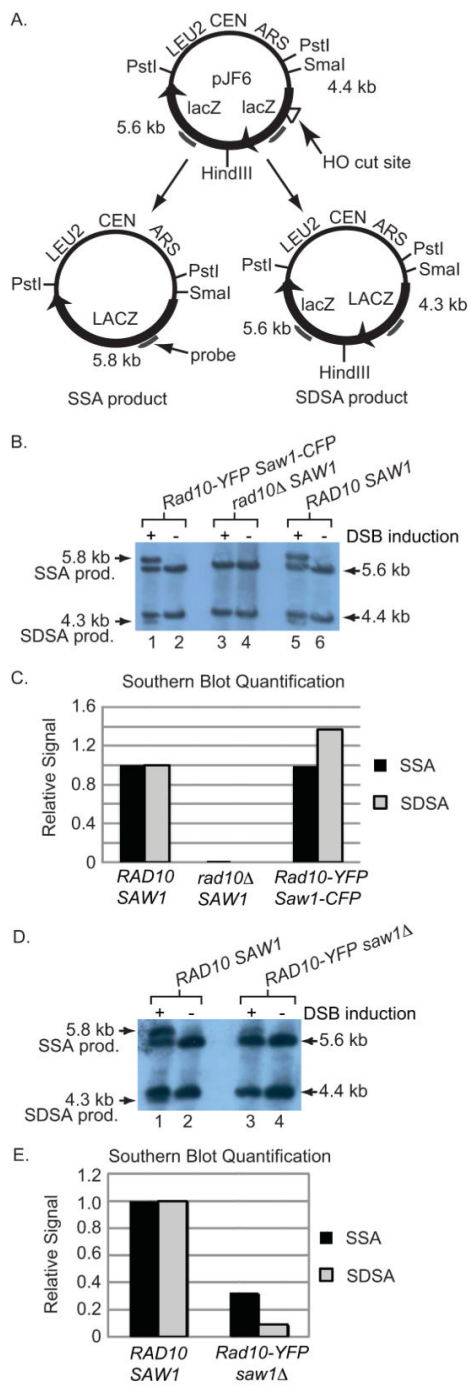

Figure 3.

Saw1-CFP is a reliable reporter of Saw1 function. A. Scheme depicting the Southern assay. Digestion by PstI, SmaI and HindIII gives 5.6 and $4.4 \mathrm{~kb}$ bands, that hybridize with the $800 \mathrm{bp} \mathrm{lacZ}$ probe. Following cleavage by HO endonuclease, the cleaved HO site can be repaired by SSA, giving a $5.8 \mathrm{~kb}$ product, or SDSA generating a $4.3 \mathrm{~kb}$ fragment. Gray lines show the position of probe hybridization. B. Southern assay using reporter plasmid pJF6. Lanes 1 and 2, strain PF149-21A, Rad10-YFP/Saw1-CFP; lanes 3 and 4, strain WPF033-6C, rad10 /SAW1; lanes 5 and 6, strain PF044-24A, RAD10/SAW1. Lanes 1, 3 and 5; galactose. Lanes 2, 4 and 6; uninduced controls. $5.8 \mathrm{kbp}$ and $4.3 \mathrm{kbp}$ repair products and 5.6 and $4.4 \mathrm{kbp}$ unrepaired products are indicated. C. Graph of densitometry scans from lanes 1, 3 and 5 in B. Data were normalized and presented as relative signal with respect to results obtained with strain PF044-24A (wild-type $R A D 10$ and $S A W 1$, lane 5, B.). D. Southern assay using reporter plasmid pJF6-HIS3. Lanes 1 and 2, strain PF044-24A, RAD10/SAWI; Lanes 3 and 4, strain PF147-35C, Rad10-YFP/saw1 L. Lanes 1 and 3; galactose. Lanes 2 and 4; uninduced controls. E. Graph of densitometry scans from lanes 1 and 3 in D. Data were treated analogously to $\mathrm{C}$. 


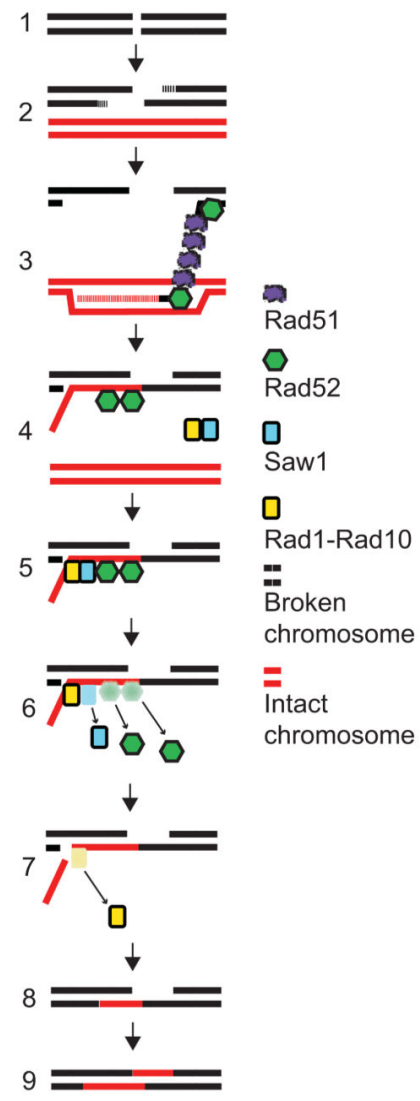

Figure 4.

Model for recruitment of Rad1-Rad10 to SDSA repair sites by Saw1. I-SceI-induced strand breaks (1) are resected (2) to give 3' single-stranded regions. Rad52 (green) and Rad51 (purple) are recruited to form synaptic filaments (3). The invading strand is copied by DNA polymerase (3) from homologous base pairing on the sister chromatid (red). After DNA synthesis, the newly synthesized strand anneals with the broken chromosome in a process mediated by Rad52 (4). A Saw1-Rad1-Rad10 complex (blue and yellow) is recruited to the flap site (5). Saw1 (blue) departs the repair site either before (as shown, 6), or possibly following flap cleavage. Rad52 departure many occur prior to, concomitant with or following Saw1 departure. The extraneous bases of the 3' flap are cleaved by Rad1-Rad10 (7), and Rad1-Rad10 departs (7), leading to gap filling by DNA polymerase and ligation of the nick (8) and gap filling of the opposite strand (9). 


\section{Table 1}

Strains used in this study.

\begin{tabular}{|c|c|c|}
\hline Name & Genotype & Where published \\
\hline PF025-7A & $\begin{array}{l}\text { MATa ade2-1 LYS2 trp1-1 can1-100 his 3-11, } 15 \text { leu2-3,112 ura3-1 TetR-mRFP (iYGL119W) } \\
\text { URA3::tetOx224(iYER187W) I-SceI (iYER186C) Rad10-YFP }\end{array}$ & [13] \\
\hline PF044-24A & MATa lys $2 \Delta$ trp $1-1$ can $1-100$ his 3-11,15 leu2-3,112 ura3-1 & {$[13]$} \\
\hline PF147-35C & $\begin{array}{l}\text { MATa ade2-1 LYS2 trp1-1 can1-100 his 3-11,15 leu2-3,112 ura3-1 TetR-mRFP (iYGL119W) } \\
\text { URA3::tetOx224(iYER187W) I-Scel (iYER186C) Rad10-YFP saw1::LEU2 }\end{array}$ & This manuscript \\
\hline PF149-21A & $\begin{array}{l}\text { MATa ade2-1 LYS2 trp1-1 can1-100 his 3-11,15 leu2-3,112 ura3-1 TetR-mRFP (iYGL119W) } \\
\text { URA3::tetOx224(iYER187W) I-Scel (iYER186C) Rad10-YFP Saw1-CFP }\end{array}$ & This manuscript \\
\hline W303-1A & MATa ade2-1 lys2 trp1-1 can1-100 his 3-11,15 leu2-3,112 ura3-1 rad5-535 & [17] \\
\hline WPF006-13C & MATa lys $2 \Delta$ trp1-1 can1-100 his 3-11,15 leu2-3,112 ura3-1 Rad10-YFP & [18] \\
\hline WPF033-6C & MATa ade2-1 rad10::KanMX his3-11,15 leu2-3,112 trp1-1 ura3-1 & [13] \\
\hline
\end{tabular}

All strains are haploid and derivatives of W303-1A and W303-1B [17]. Additionally, all strains are wild-type for the ADE2 and RAD5 genes unless otherwise noted. 